\title{
Don't Waste a Crisis: Opportunities to Enhance BME Student Learning Through COVID-19
}

\author{
Mark C. Pierce (10) \\ Department of Biomedical Engineering, Rutgers, The State University of New Jersey, New Brunswick, NJ, USA
}

(Received 30 June 2020; accepted 18 August 2020; published online 8 September 2020)

\section{CHALLENGE STATEMENT}

Maintaining student engagement and motivation can be challenging with remote teaching. Hererra et al. ${ }^{3}$ described several contributing factors, including the need to maintain strong communication and meaningful interactions between students and instructors, supporting a learning community among students, and difficulties in use of technology. In seeking to identify activities and interactions that can mitigate these challenges, Dixson et $a ._{.}^{2}$ proposed the application of concepts to case studies, use of discussion forums, research papers, and assignments on current events. The COVID-19 pandemic is a public health emergency that continues to affect the lives of students and their families in unprecedented ways. This article highlights how contemporary topics associated with the COVID-19 pandemic can be integrated into coursework across the biomedical engineering curriculum.

\section{NOVEL INITIATIVE}

Many engineering courses try to connect theoretical topics with practical applications to stimulate student interest and engagement. COVID-19 provides educators with a unique opportunity to demonstrate how training in biomedical engineering can have immediate and real-world impact on public health. The Department of Biomedical Engineering at Rutgers University offers an elective course on Biomedical Engineering for

Address correspondence to Mark C. Pierce, Department of Biomedical Engineering, Rutgers, The State University of New Jersey, New Brunswick, NJ, USA. Electronic mail: mark.pierce@ rutgers.edu
Global Health. This is a 3-credit course open to seniors majoring in biomedical engineering, meeting for two 80-min lectures each week. In Spring 2020, eighty-seven students completed the course. The first 7 weeks used conventional in-person instruction while the second 7 weeks switched to remote instruction, with synchronous lectures recorded and posted on Canvas for asynchronous access. Compared to previous offerings, approximately $25 \%$ of the overall class time was dedicated to reviewing the latest scientific data and clinical evidence on COVID-19. Students were encouraged to identify and discuss material encountered during their own reading on the subject. The instructor also introduced peer-reviewed journal publications, guidance from public health institutes and agencies, and articles from print and online news media (Supplementary Materials, S1). A diverse range of sources and perspectives was chosen to illustrate the breadth of expertise required to tackle a major public health challenge and to highlight the role of biomedical engineers within this ecosystem. Course assessments shifted to focus on COVID-19 as well to further motivate students to keep up to date on developments.

Identification of challenges for biomedical engineers often begins with analysis of epidemiologic data at the local, regional, or national level. Incidence, prevalence, and mortality figures can inform us on priority diseases and conditions to focus on. As the COVID-19 pandemic began to develop, students reviewed and discussed incidence and mortality figures from sources including the Johns Hopkins University COVID-19 Dashboard and The COVID Tracking Project. Data at the state and county level from the New Jersey Dept. of Health was particularly compelling as students could directly see the impact of the pandemic in their home areas. Reviewing these data also allowed for discussions on how to interpret and effectively present data, including the appropriate use of linear or logarithmic 
scales, sliding window averaging, and time-shifting data series to compare plots.

This understanding of current and historical data on COVID-19 naturally led into the topic of modeling the future trajectory of the pandemic. Students discussed weekly updates from models including those from the Institute of Health Metrics and Evaluation, relating the advanced statistical techniques used to basic approaches that they had previously encountered in the core BME curriculum, based on systems of first order linear ordinary differential equations.

Diagnostic testing for COVID-19 ramped up in the United States during the month of March. Students described their own experiences with nasal swab collection. This provided strong motivation to understand the fundamental concepts behind these nucleic acid based tests for viral RNA and how to interpret test results. Lectures first highlighted the critical role of genetic sequencing techniques and tools covered in bioinformatics courses, such as the NCBI GenBank databases for identifying target sequences. The RTPCR methods that most COVID-19 tests employ were examined and related to students' prior experience performing PCR in the laboratory component of their core biology-I course. Novel isothermal amplification techniques that offer new capabilities for point-of-care nucleic acid testing brought students to the cutting edge of this field. Beyond studying the scientific principles underlying these tests, students also developed the ability to interpret results through quantitative metrics including sensitivity, specificity, predictive values, and likelihood ratios. Soon after these viral testing capabilities became established, serologic (antibody) testing began to emerge, providing critical information on the true prevalence of COVID-19 and potential immunity. These diagnostic tests were related to the BME measurements lab course where students previously performed hands-on ELISA reactions to learn about protein detection.

The next key topic to gain the public's attention was the prospect of a vaccine against COVID-19. To appreciate the challenges in developing any vaccine, a basic understanding of immunology is required. About half of the class had some exposure to basic immunology through taking biology-II as an elective course. A lecture refreshing adaptive immunity and immunologic memory was sufficient for most students to grasp the key principles of vaccination. Students could remember specific vaccines they had received themselves but were unaware of the traditional and novel techniques used to produce vaccines. This topic exposed students to novel bioengineering techniques including mRNA/DNA vaccines and gene therapy.

Beyond highlighting these technologies aimed at tackling COVID-19, there were opportunities to examine the challenges faced in bringing biomedical technologies to the market through clinical trials and regulatory review, subjects also encountered in their capstone design course. Students learned about the role of the US Food and Drug Administration (FDA) in reviewing diagnostic tests, including the use of emergency use authorization (EUA) to advance technology in the public interest during exceptional circumstances. The urgent quest for a COVID-19 vaccine enabled the traditional multi-phase clinical trial sequence to be compared with new paradigms that perform multiple phases in parallel. This topic also led to stimulating discussions over ethics, including proposals for human challenge studies to accelerate vaccine development, and how to achieve equitable distribution of an effective vaccine. Ongoing controversies surrounding COVID-19 treatment notwithstanding, students learned about the existence and importance of expert panel guidance on making recommendations for therapeutic interventions. The emergence of candidate drugs provided opportunities to focus on methods for experimental design and hypothesis testing, which brought to life key concepts from statistics courses including study design, sample size, significance, and power.

While there are many opportunities to use COVID19 to highlight technical concepts in biomedical engineering, there are also ways to show students how their skills could positively impact the ongoing pandemic and to think about future career paths in our field. In this Spring 2020 course, students were genuinely motivated by seeing efforts by our own institution's research community having significant impact in the community. These initiatives included 3D printing of personal protective equipment for healthcare workers, development of novel point-of-care diagnostic tests for COVID-19, and clinical trials on the use of convalescent plasma treatment.

Beyond integration of COVID-19 themes within lectures, the course also used homework assignments, exams, and a project to encourage independent study on the pandemic. One homework assignment required students to review an article in Science Magazine on vaccine candidates and progress of early clinical trials (Supplementary Materials, S3). The final exam required students to read a journal article ${ }^{4}$ on a new diagnostic test for SARS-CoV-2 that had been published only 1 week earlier, emphasizing to students that the material and concepts they were learning in class were directly applicable to the latest cutting-edge research on COVID-19 (Supplementary Materials, S4). Students also completed a project assignment where they had to write a two-page proposal for a new COVID-19 diagnostic test (modeled after the Bill and Melinda Gates Grand Challenges program) (Supple- 
mentary Materials, S5). Students were given plenty of latitude and room for creativity but were also provided with an organizing structure for guidance, breaking down the proposal into Background, Approach, and Evaluation sections. This project assignment generated many creative ideas and compelled students to dive into the rapidly expanding literature on diagnostics.

\section{REFLECTION}

As instructors, we hope that students will apply the material covered in our classes, but it is rare that an opportunity presents itself to learn about a topic as it unfolds in real-time. In previous semesters, students would learn about malaria and tuberculosis, ancient infectious diseases which still have high mortality rates in other parts of the world but no meaningful impact on their own lives. When discussing HIV, students would appreciate how little was known about the pathophysiology and transmissibility of the disease in the 1980 s, before decades of basic and clinical research taught us how to effectively treat and manage the disease. With COVID-19, students saw new knowledge emerge and be put into practice within the course of a single semester. They also saw how missteps in public health policy and technology developments led to setbacks. In the course evaluation survey, many students alluded to valuing the sense of learning new material on topics that were impacting their own daily lives. Students also felt empowered by the project assignment, challenging them to propose a new or improved diagnostic test for COVID-19. These observations are consistent with prior research on students' perceptions about challenge-based instruction ${ }^{1,5}$ and engaging BME students to solve major global health problems. ${ }^{6}$ In future courses, student motivation could be measured using a tool like Dixson's "online student engagement scale", ${ }^{2}$ or an alternative survey tailored to the mode of instruction and pedagogical question(s) being asked. Learning outcomes could be measured by comparing scores in homework, exam, and project submissions on contemporary topics like COVID-19, to assignments on topics covered retrospectively. Students' own perception of their mastery of the material could be captured through the use of course evaluation surveys.

Difficulties encountered with this approach include providing all students with an opportunity to contribute to discussions on COVID-19, particularly with a large class size. Keeping discussions centered on reliable sources of information also required continu- ous effort as students would sometimes relay anecdotal stories or claims with little scientific basis. Approaches that helped included having the instructor regularly introduce and post reading material from reliable sources to serve as examples (Supplementary Materials, S1). During discussions, encouraging other students to offer alternate perspectives and moderate the conversation proved more effective than simply having the instructor comment directly on a student's opinion. Other challenges with teaching a topic that is evolving in real-time include dedicating preparation time to keep up with and distill new information, even on the same day as a lecture. It is important also to reassure students that theories can change as new knowledge emerges, such as treatment guidelines or input assumptions for modeling.

The COVID-19 pandemic will continue to impact us over the next several semesters. But we can be hopeful that vaccines will emerge from clinical trials, rapid diagnostic testing will be scaled up and deployed for at-home use, digital technologies for contact tracing will be widely adopted, and treatment strategies will be elucidated. These developments all involve concepts from biomedical engineering that can be highlighted in our courses, potentially inspiring students to develop and apply their skills and energy to overcome this ongoing pandemic.

\section{ELECTRONIC SUPPLEMENTARY MATERIAL}

The online version of this article (https://doi.org/10. 1007/s43683-020-00021-0) contains supplementary material, which is available to authorized users.

\section{AUTHOR CONTRIBUTIONS}

MCP: All contributions.

\section{FUNDING}

Not applicable.

AVAILABILITY OF DATA AND MATERIAL

Supplementary materials are provided.

\section{CONFLICT OF INTEREST}

Not applicable. 
Not applicable.

\section{ETHICS APPROVAL}

\section{CONSENT TO PARTICIPATE}

Not applicable.

\section{CONSENT FOR PUBLICATION \\ Not applicable.}

\section{REFERENCES}

${ }^{1}$ Clegg JR, Diller KR. Development and transfer of innovative problem solving strategies and related confidence in biomedical engineering. In: ASEE Gulf-Southwest Section Annual Meeting 2018 Papers. American Society for Engineering Education. https://peer.asee.org/31565.

${ }^{2}$ Dixson MD. Creating effective student engagement in online courses: What do students find engaging? J Scholarship Teach Learn. 2010;10(2):1-13.
${ }^{3}$ Herrera LMM, Valenzuela JCM. What kind of teacher achieves student engagement in a synchronous online model? In 2019 IEEE Global Engineering Education Conference (EDUCON) (pp. 227-231). IEEE.

${ }^{4} \mathrm{Lu} \mathrm{R}$, Wu X, Wan Z, Li Y, Jin X, Zhang C. A novel reverse transcription loop-mediated isothermal amplification method for rapid detection of SARS-CoV-2. Int J Mol Sci. 2020;21(8):2826.

${ }^{5}$ Martin T, Rivale SD, Diller KR. Comparison of student learning in challenge-based and traditional instruction in biomedical engineering. Ann Biomed Eng. 2007;35(8):131223.

${ }^{6}$ Oden M, Mirabal Y, Epstein M, Richards-Kortum R. Engaging undergraduates to solve global health challenges: a new approach based on bioengineering design. Ann Biomed Eng. 2010;38(9):3031-41.

Publisher's Note Springer Nature remains neutral with regard to jurisdictional claims in published maps and institutional affiliations. 